Research Article

\title{
Boundedly Rational User Equilibrium with Restricted Unused Routes
}

\author{
Chao Sun, ${ }^{1}$ Menghui $\mathrm{Li}^{2}{ }^{2}$ Lin Cheng, ${ }^{1}$ Senlai $\mathrm{Zhu}^{1}{ }^{1}$ and Zhaoming $\mathrm{Chu}^{3}$ \\ ${ }^{1}$ School of Transportation, Southeast University, Nanjing 210096, China \\ ${ }^{2}$ School of Highway, Chang'an University, Xian 710064, China \\ ${ }^{3}$ Road Traffic Safety Research Center of the Ministry of Public Security, Beijing 100062, China \\ Correspondence should be addressed to Lin Cheng; gist@seu.edu.cn
}

Received 11 July 2016; Revised 30 September 2016; Accepted 6 November 2016

Academic Editor: Cengiz Çinar

Copyright (c) 2016 Chao Sun et al. This is an open access article distributed under the Creative Commons Attribution License, which permits unrestricted use, distribution, and reproduction in any medium, provided the original work is properly cited.

\begin{abstract}
A boundedly rational user equilibrium model with restricted unused routes (R-BRUE) considering the restrictions of both used route cost and unused route cost is proposed. The proposed model hypothesizes that for each OD pair no traveler can reduce his/her travel time by an indifference band by unilaterally changing route. Meanwhile, no route is unutilized if its travel time is lower than sum of indifference band and the shortest route cost. The largest and smallest used route sets are defined using mathematical expression. We also show that, with the increase of the indifference band, the largest and smallest used route sets will be augmented, and the critical values of indifference band to augment these two path sets are identified by solving the mathematical programs with equilibrium constraints. Based on the largest and smallest used route sets, the R-BRUE route set without paradoxical route is generated. The R-BRUE solution set can then be obtained by assigning all traffic demands to the corresponding generated route set. Various numerical examples are also provided to illustrate the essential ideas of the proposed model and structure of R-BRUE route flow solution set.
\end{abstract}

\section{Introduction}

Perfect rationality is widely used in studying traditional transportation network models in which traveler always chooses the shortest (i.e., least utility) route, such as user equilibrium (UE [1]) and stochastic user equilibrium (SUE [2]) traffic assignment models. However, travelers may not always choose shortest route due to (1) lack of perfect travel information; (2) incapability of obtaining the shortest route with the complex traffic situations; and (3) certain "inertia" in decision making. Therefore people do not always choose the route with the maximum utility. They tend to seek a satisfactory route instead.

In the literature of evaluating habitual routes in route choice behavior, only $30 \%$ of respondents from Boston [3], $59 \%$ from Cambridge, Massachusetts [4], and 86.8\% from Turin, Italy [5] chose the shortest routes. Based on GPS studies, Zhu [6] found that $90 \%$ of subjects in the MinneapolisSt. Paul region choose routes one-fifth longer than average commute time. All findings above revealed that people do not usually take the shortest routes and the used routes generally have higher costs than shortest ones.

It is more practical that traveler is boundedly rational (BR); traveler will not change his/her route if his/her travel time is a little longer than the shortest route. A series of experiments were conducted to empirically validate bounded rationality [7-13]. The results showed that, in the repeated learning process, commuters would not change their routes unless the difference between preferred arrival time and actual arrival time exceeded a threshold. And boundedly rational route choice modeling observed from experiments provided a valid description of actual commuter daily behavior.

Simon, in 1957 [14], first proposed the notion of bounded rationality. And in 1987 [7], Mahmassani and Chang introduced it to traffic modeling. Since then, bounded rationality has received considerable attention in various transportation models, such as traffic safety [15], transportation planning [16, 17], traffic policy making [18-20], traffic assignment, and network design [21-28]. All these studies indicated that traveler is boundedly rational in his/her decision-making process. 
Boundedly rational user equilibrium (BRUE) is a network state such that travelers can take any route whose travel time is within a threshold of the shortest route time [22, 23, 2528]. Such a threshold is phrased by Mahmassani and Chang [7] as "indifference band." In other words, no one can reduce his/her travel time by an indifference band by unilaterally changing his/her route. The indifference band is estimated from either laboratory experiment data or a behavioral study of road users (e.g., by surveys $[7,29])$. By introducing indifference band for each OD pair, the BRUE relaxes UE assumption that travelers only take the shortest routes at equilibrium.

However, unlike the conventional UE model, the traffic flow under BRUE may not utilize any shortest or least-cost route; in another word the unused route cost may be lower than the used one. For example, the route flows are 0, 5, and 7 for three different routes on one OD pair, and the travel times are 10,12 , and 13 , respectively. If the indifference band is 3 , the above route flow solution is a BRUE solution. From the behavioral point of view, one might question the plausibility of this that the least travel time route has no traffic on it. Therefore, to make the model become behavioral more defensible, not only the used route cost should be restricted, but also the unused route cost should be restricted.

Uncertainties are unavoidable in transportation systems and make people become boundedly rational. Travelers do not know exactly the time that they arrive at the destination due to the travel time variability which is made by uncertainties. However, in many cases (such as going to work, having a meeting, and catching the train), travelers care more about arrive time than travel time; no one wants to be late; thus the largest and smallest route sets exist in travels' trip process.

This paper makes contributions in three major areas: (1) considering both the restrictions of used route cost and unused route cost in the route decision process, we present a boundedly rational user equilibrium model with restricted unused routes (R-BRUE). This new model hypothesizes that for each OD pair no traveler can reduce his/her travel time by an indifference band by unilaterally changing route. Meanwhile, no route is unutilized if its travel time is lower than sum of indifference band and the shortest route cost. (2) We propose the largest and smallest used route sets which can be used to generate the used route set of R-BRUE model. These two used route sets are defined as the union and intersection of all R-BRUE solution set patterns, respectively. And (3) we develop two mathematical programs (MP) with equilibrium constraint to solve the critical values which is used to augment the largest and smallest used route sets.

The remainder of the paper is organized as follows. In Section 2, $\boldsymbol{\varepsilon}$-R-BRUE ( $\boldsymbol{\varepsilon}$ denotes the indifference band) is defined and its mathematical formulation is established. In Section 3, the largest and smallest $\boldsymbol{\varepsilon}$-used route sets are defined, and their properties are studied. In Section $4, \boldsymbol{\varepsilon}$-RBRUE route set without paradoxical route is generated. In Section 5, $\boldsymbol{\varepsilon}$-R-BRUE route flow set without paradoxical route is constructed, and some examples are presented to illustrate the essential ideas of proposed model and the structure of RBRUE route flow solution set. Finally, some conclusions and future work are provided.

\section{Definition of $\varepsilon$-R-BRUE and Mathematical Formulation}

In this section, we propose $\boldsymbol{\varepsilon}$-boundedly rational user equilibrium model with restricted unused routes (R-BRUE) and the mathematical formulation of proposed model.

2.1. Definition of $\boldsymbol{\varepsilon}-R$-BRUE. Consider a transportation network $G=[N, A]$, where $N$ and $A$ denote the sets of nodes and links, respectively. Let $W$ denote the set of OD pairs for which travel demand $q^{\omega}$ is generated between OD pair $\omega \in W$, and let $f_{k}^{\omega}$ denote the traffic flow on route $k \in K^{\omega}$, where $K^{\omega}$ is the set of routes connecting OD pair $\omega$ and all $K^{\omega}$ constitute $K$. The feasible route flow set is to assign the traffic demand on the feasible routes: $F \triangleq\left\{\mathbf{f}: \mathbf{f} \geq 0, \sum_{k \in K^{\omega}} f_{k}^{\omega}=q^{\omega}, \forall \omega \epsilon\right.$ $W$ \}. Below is formal definition of $\boldsymbol{\varepsilon}$-boundedly rational user equilibrium with restricted unused routes (R-BRUE).

Definition 1. $\boldsymbol{\varepsilon}$-boundedly rational user equilibrium with restricted unused routes ( $\boldsymbol{\varepsilon}$-R-BRUE) is a network state such that the travel cost of all used route is less than or equal to the sum of given indifference band $\varepsilon=\left(\varepsilon^{\omega}\right)_{\omega \in W} \geq 0$ and the shortest route cost; meanwhile, the travel cost of the unused route is greater than or equal to the sum of $\boldsymbol{\varepsilon}$ and the shortest route cost; that is,

$$
\begin{aligned}
& f_{k}^{\omega}>0 \Longrightarrow \\
& C_{k}^{\omega}(\mathbf{f}) \leq \min _{i \in K^{\omega}} C_{i}^{\omega}(\mathbf{f})+\varepsilon^{\omega}, \\
& f_{k}^{\omega}=0 \Longrightarrow \quad \forall k \in K^{\omega}, \omega \in W, \\
& C_{k}^{\omega}(\mathbf{f}) \geq \min _{i \in K^{\omega}} C_{i}^{\omega}(\mathbf{f})+\varepsilon^{\omega}, \\
& \forall k \in K^{\omega}, \omega \in W,
\end{aligned}
$$

where $\mathbf{f}$ is the vector form of traffic flow $f_{k}^{\omega}: \mathbf{f}=\left(\ldots, f_{k}^{\omega}\right.$, $\ldots)^{T}$ and $C_{k}^{\omega}(\cdot)$ is the route cost function on route $k$ between OD pair $\omega$.

We should point out that boundedly rational user equilibrium (BRUE) model only considers $f_{k}^{\omega}>0 \Rightarrow C_{k}^{\omega}(\mathbf{f}) \leq$ $\min _{i \in K^{\omega}} C_{i}^{\omega}(\mathbf{f})+\varepsilon^{\omega}$ which do not take the cost of unused route into consideration. We first use $f_{k}^{\omega}=0 \Rightarrow C_{k}^{\omega}(\mathbf{f}) \geq$ $\min _{i \in K^{\omega}} C_{i}^{\omega}(\mathbf{f})+\varepsilon^{\omega}$ to restrict the "irrational solutions."

Equation (1) gives a necessary condition judging whether a flow pattern is R-BRUE and is equivalent to the following condition:

$$
\begin{aligned}
C_{k}^{\omega}(\mathbf{f}) & >\min _{i \in K^{\omega}} C_{i}^{\omega}(\mathbf{f})+\varepsilon^{\omega} \Longrightarrow \\
f_{k}^{\omega} & =0, \\
C_{k}^{\omega}(\mathbf{f}) & <\min _{i \in K^{\omega}} C_{i}^{\omega}(\mathbf{f})+\varepsilon^{\omega} \Longrightarrow \\
f_{k}^{\omega} & >0 .
\end{aligned}
$$

In other words, a used route has lower cost than an unused one, which is the same as that in the UE (user equilibrium) 
setting. When $\varepsilon^{\omega}=0$ for each $\omega$, the R-BRUE definition is reduced to the UE problem.

Theorem 2. Any $\varepsilon$-R-BRUE solution is also a $\boldsymbol{\varepsilon}$-BRUE solution. $\boldsymbol{\varepsilon}$-BRUE solution may not, however, necessarily fulfill $\boldsymbol{\varepsilon}$ $R$-BRUE conditions.

Proof. let $\mathbf{f}$ be a route flow pattern to $\boldsymbol{\varepsilon}$-R-BRUE model. Then, for $f_{k}^{\omega}>0, C_{k}^{\omega}(\mathbf{f}) \leq \min _{i \in K^{\omega}} C_{i}^{\omega}(\mathbf{f})+\varepsilon^{\omega}$ hold for all $k \in K^{\omega}$ and $\omega \in W$; that is, $f_{k}^{\omega}>0 \Rightarrow C_{k}^{\omega}(\mathbf{f}) \leq \min _{i \in K^{\omega}} C_{i}^{\omega}(\mathbf{f})+\varepsilon^{\omega}$, $\forall k \in K^{\omega}, \omega \in W$, which satisfies $\boldsymbol{\varepsilon}$-BRUE model.

For the converse situation, suppose that a flow allocation satisfies $\boldsymbol{\varepsilon}$-BRUE conditions and in addition has an unused route which has a cost less than the sum of $\varepsilon$ and the shortest route. Then $\boldsymbol{\varepsilon}$-R-BRUE conditions are violated.

Usually $\boldsymbol{\varepsilon}$-R-BRUE is nonunique. Denote a set containing all route flow patterns satisfying Definition 1 as $\boldsymbol{\varepsilon}$-R-BRUE route flow solution set:

$$
\begin{aligned}
& F_{\mathrm{R}-\mathrm{BRUE}}^{\varepsilon} \triangleq\left\{\mathbf{f} \in F: f_{k}^{\omega}>0 \Longrightarrow C_{k}^{\omega}(\mathbf{f}) \leq \min _{i \in K^{\omega}} C_{i}^{\omega}(\mathbf{f})\right. \\
& +\varepsilon^{\omega}, f_{k}^{\omega}=0 \Longrightarrow C_{k}^{\omega}(\mathbf{f}) \geq \min _{i \in K^{\omega}} C_{i}^{\omega}(\mathbf{f})+\varepsilon^{\omega}, \forall k \\
& \left.\quad \in K^{\omega}, \omega \in W\right\} .
\end{aligned}
$$

Theorem 3. If the link cost function is continuous, $\boldsymbol{\varepsilon}-R-B R U E$ solution $(\varepsilon \geq 0)$ is nonempty.

Proof. First, Patriksson [30] showed that, when the link cost function is continuous, UE solution exists. Let $\mathbf{f}_{\mathrm{UE}} \in F_{\mathrm{UE}}$ be one UE route flow pattern, and set $\mathbf{f}_{1} \triangleq\left\{f_{i}: f_{i} \in \mathbf{f}_{\mathrm{UE}}, f_{i}>0\right\}$, $\mathbf{f}_{0} \triangleq\left\{f_{i}+\delta: f_{i} \in \mathbf{f}_{\mathrm{UE}}, f_{i}=0, C_{k}^{\omega}\left(f_{i}\right)<\min _{i \in K^{\omega}} C_{i}^{\omega}\left(\mathbf{f}_{\mathrm{UE}}\right)+\varepsilon^{\omega}\right\}$, where $\delta$ is a very small positive parameter. Let $\mathbf{f}=\left[\mathbf{f}_{0}, \mathbf{f}_{1}\right]$, when $\boldsymbol{\varepsilon} \geq 0$,

$$
\begin{aligned}
f_{k}^{\omega} & >0 \Longrightarrow \\
C_{k}^{\omega}(\mathbf{f}) & <\min _{i \in K^{\omega}} C_{i}^{\omega}(\mathbf{f})+\varepsilon^{\omega} \leq \min _{i \in K^{\omega}} C_{i}^{\omega}(\mathbf{f})+\varepsilon^{\omega}, \\
f_{k}^{\omega} & =0 \Longrightarrow \\
C_{k}^{\omega}(\mathbf{f}) & \geq \min _{i \in K^{\omega}} C_{i}^{\omega}(\mathbf{f})+\varepsilon^{\omega},
\end{aligned}
$$

$$
\forall k \in K^{\omega}, \omega \in W .
$$

So $\mathbf{f}$ is $\boldsymbol{\varepsilon}$-R-BRUE solution $(\boldsymbol{\varepsilon} \geq 0)$; that is, $\mathbf{f} \in F_{\mathrm{R} \text {-BRUE }}^{\boldsymbol{\varepsilon}}$. Given the continuous link cost function, at least one $\boldsymbol{\varepsilon}$-R-BRUE flow pattern exists, and therefore $F_{\mathrm{R} \text {-BRUE }}^{\varepsilon} \neq \varnothing$.

2.2. R-BRUE Mathematical Formulation. We use slack variables $\rho_{k}^{\omega}$ to define R-BRUE mathematically. $\mathbf{f}$ is a R-BRUE distribution if and only if there exists $\pi^{\omega}$ whose physical meaning is the minimum route cost for every $\omega$ such that

$$
\begin{gathered}
C_{k}^{\omega}(\mathbf{f})-\pi^{\omega}-\rho_{k}^{\omega}=0, \quad \forall k \in K^{\omega}, \omega \in W, \\
f_{k}^{\omega}\left(\varepsilon^{\omega}-\rho_{k}^{\omega}\right)\left\{\begin{array}{ll}
=0, & \text { if } \varepsilon^{\omega} \leq \rho_{k}^{\omega}, \\
>0, & \text { o.w., }
\end{array} \quad \forall k \in K^{\omega}, \omega \in W,\right.
\end{gathered}
$$

$$
\begin{gathered}
\sum_{k \in K^{\omega}} f_{k}^{\omega}=q^{\omega}, \quad \forall \omega \in W, \\
f_{k}^{\omega} \geq 0, \quad \forall k \in K^{\omega}, \omega \in W, \\
\rho_{k}^{\omega} \geq 0, \quad \forall k \in K^{\omega}, \omega \in W,
\end{gathered}
$$

where $q^{\omega}$ is the traffic demand between OD pair $\omega$. Note that when $\varepsilon=0$ for all $\omega, 2.2$ reduces to $0 \leq f_{k}^{\omega} \perp C_{k}^{\omega}(\mathbf{f})-\pi^{\omega} \geq 0$, $\sum_{k \in K^{\omega}} f_{k}^{\omega}=q^{\omega}$ which is the conventional UE conditions.

\section{Largest and Smallest $\varepsilon$-Used Route Sets}

In this section, we give the definition of the largest and smallest $\boldsymbol{\varepsilon}$-used route sets, and we also discuss the properties of two proposed route sets.

3.1. Definitions. Here we give three definitions of used route set, largest $\boldsymbol{\varepsilon}$-used route set ( $\boldsymbol{\varepsilon}$-URS) and smallest $\boldsymbol{\varepsilon}$-URS of $\boldsymbol{\varepsilon}$-R-BRUE model as follows.

Definition 4. Given $\boldsymbol{\varepsilon}$-R-BRUE route pattern $\mathbf{f} \in F_{\mathrm{R}-\mathrm{BRUE}}^{\boldsymbol{\varepsilon}}$, the used route carries flow, while its travel cost is within the shortest cost plus the indifference band; that is,

$$
\begin{aligned}
& a^{\varepsilon}(\mathbf{f})=\left\{k \in K^{\omega}: f_{k}>0, C_{k}^{\omega}(\mathbf{f}) \leq \min _{i \in K^{\omega}} C_{i}^{\omega}(\mathbf{f})\right. \\
& \left.+\varepsilon^{\omega}, \forall \omega \in W\right\} .
\end{aligned}
$$

Definition 5. A largest $\boldsymbol{\varepsilon}$-URS contains all used routes for every flow pattern in $\boldsymbol{\varepsilon}$-R-BRUE flow set, mathematically:

$$
K_{l}^{\varepsilon}=\bigcup_{\mathbf{f} \in F_{\mathrm{R}-\mathrm{BRUE}}^{\varepsilon}} a^{\varepsilon}(\mathbf{f}) .
$$

Definition 6. A smallest $\boldsymbol{\varepsilon}$-URS contains the used routes which all flow patterns in $\varepsilon$-R-BRUE flow set have, mathematically:

$$
K_{s}^{\varepsilon}=\bigcap_{\mathbf{f} \in F_{\mathrm{R}-\mathrm{BRUE}}^{\varepsilon}} a^{\varepsilon}(\mathbf{f})
$$

3.2. Monotonically Nondecreasing Largest $\boldsymbol{\varepsilon}$-URS. In the following, we will discuss the impact of the value of $\varepsilon$ on the size of the largest $\boldsymbol{\varepsilon}$-URS.

Theorem 7. If $0 \leq \boldsymbol{\varepsilon}<\boldsymbol{\varepsilon}^{\prime}$, then $K_{l}^{\varepsilon} \subseteq K_{l}^{\varepsilon^{\prime}}$, where $K_{l}^{\varepsilon}$ is defined in (7).

Proof. Let $\mathbf{f}^{\varepsilon} \in F_{\mathrm{M} \text {-BRUE }}^{\boldsymbol{\varepsilon}}$ be one $\boldsymbol{\varepsilon}$-R-BRUE route flow pattern, and set $\mathbf{f}_{1} \triangleq\left\{f_{i}: f_{i} \in \mathbf{f}^{\varepsilon}, f_{i}>0\right\}, \mathbf{f}_{0} \triangleq\left\{f_{i}+\delta: f_{i} \in\right.$ $\left.\mathbf{f}^{\varepsilon}, f_{i}=0, C_{k}^{\omega}\left(f_{i}\right)<\min _{i \in K^{\omega}} C_{i}^{\omega}\left(\mathbf{f}^{\varepsilon}\right)+\boldsymbol{\varepsilon}^{\prime}\right\}$, where $\delta$ is a very small positive parameter. Let $\mathbf{f}^{\varepsilon^{\prime}}=\left[\mathbf{f}_{0}, \mathbf{f}_{1}\right]$; then

$$
\begin{aligned}
f_{k}^{\omega} & >0 \Longrightarrow \\
C_{k}^{\omega}(\mathbf{f}) & <\min _{i \in K^{\omega}} C_{i}^{\omega}(\mathbf{f})+\varepsilon^{\omega^{\prime}} \leq \min _{i \in K^{\omega}} C_{i}^{\omega}(\mathbf{f})+\varepsilon^{\omega^{\prime}},
\end{aligned}
$$




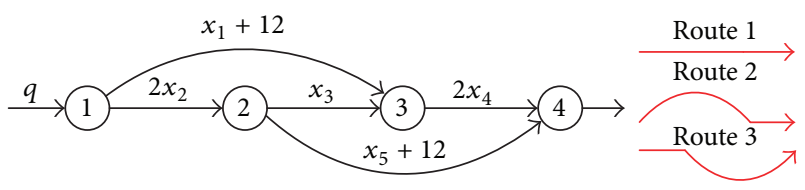

FIgURE 1: Test network and routes characteristics.

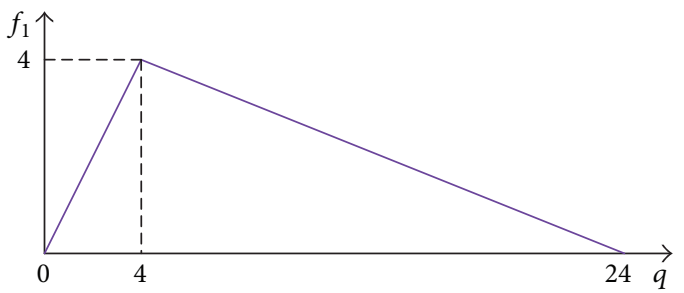

FIGURE 2: The UE traffic flow of route 1 under different demand.

$$
\begin{aligned}
f_{k}^{\omega} & =0 \Longrightarrow \\
C_{k}^{\omega}(\mathbf{f}) & \geq \min _{i \in K^{\omega}} C_{i}^{\omega}(\mathbf{f})+\varepsilon^{\omega^{\prime}},
\end{aligned}
$$

$\forall k \in K^{\omega}, \omega \in W$

So $\mathbf{f}^{\boldsymbol{\varepsilon}^{\prime}}$ is $\boldsymbol{\varepsilon}^{\prime}$-R-BRUE solution, and $a^{\boldsymbol{\varepsilon}}(\mathbf{f}) \subseteq a^{\boldsymbol{\varepsilon}^{\prime}}$ (f). Based on (7), we can get that $K_{l}^{\varepsilon} \subseteq K_{l}^{\varepsilon^{\prime}}$.

3.3. Paradoxical Route and Property of Smallest $\boldsymbol{\varepsilon}$-URS. We will give the definition of paradoxical route first.

Definition 8. With the increasing of OD demand, the traffic flow on route $p$ first increases and then decreases at the UE state. And the route $p$ is defined as a paradoxical route.

This phenomenon contradicts our intuition. Following that, we use an example to illustrate this paradox.

The network topology of the test network, link, and traffic demand characteristics are depicted in Figure 1. The network consists of 4 nodes, 5 links, and 1 OD pair. Red curves on the right indicate 3 routes.

The equilibrium route flow $f_{1}$ by varying the traffic demand $q$ from zero to infinity is shown in Figure 2. When the traffic demand is lower than 4 , all the travelers pick route 1 ; and $f_{1}$ is increasing with the increasing of demand. While $q$ reached to $4, f_{1}$ is decreasing with the increasing of $q$. And when the demand reaches to 24 , the flow on route 1 becomes 0 . Based on Definition 8 , we can get that route 1 is the paradoxical route.

When $0 \leq \boldsymbol{\varepsilon}<\boldsymbol{\varepsilon}^{\prime}, K_{s}^{\varepsilon} \subseteq K_{s}^{\varepsilon^{\prime}}$, where the network contains paradoxical route, monotonically nondecreasing property of smallest $\boldsymbol{\varepsilon}$-URS may not be satisfied due to exiting paradoxical route in the network. Consider the test network in Figure 3 which consists of 4 nodes, 6 links, and 1 OD pair. The link travel time functions and traffic demand are reported in Figure 3. Red curves on the right indicate 4 routes.

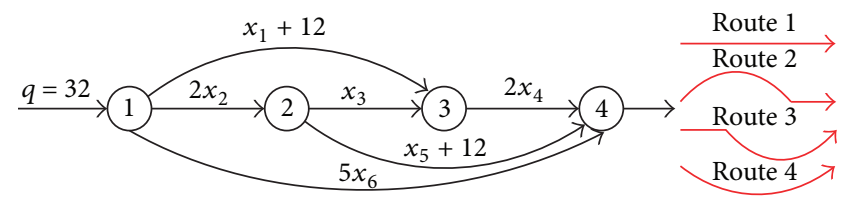

FIgURE 3: Test network and routes characteristics.

Solving the UE where $\varepsilon=0$ for the R-BRUE, we have $f_{1}=$ $0.25, f_{2}=11.25, f_{3}=11.25$, and $f_{4}=9.25$. Therefore, the smallest $\boldsymbol{\varepsilon}$-URS is $K_{s}^{0}=\{1,2,3,4\}$. When $\varepsilon=48, f_{1}=0, f_{2}=$ $15, f_{3}=15$, and $f_{4}=2$, the travel time is $C_{1}=60, C_{2}=57$, $C_{3}=57$, and $C_{4}=10$. The minimum OD travel time is 10 and all the utilized routes have travel times of no more than $10+$ $48=58$. Therefore, the route flow pattern is a valid R-BRUE flow. However, the smallest $\boldsymbol{\varepsilon}$-URS which is $K_{s}^{48}=\{2,3,4\}$ does not satisfy the monotonically nondecreasing property.

Theorem 9 (monotonically nondecreasing smallest $\boldsymbol{\varepsilon}$-URS without paradoxical route). If $0 \leq \boldsymbol{\varepsilon}<\boldsymbol{\varepsilon}^{\prime}$, then $K_{s}^{\varepsilon} \subseteq K_{s}^{\varepsilon^{\prime}}$ for a network without paradoxical route, where $K_{s}^{\varepsilon}$ is defined in (8).

Proof. Assume that there exist two bands $\boldsymbol{\varepsilon}$ and $\boldsymbol{\varepsilon}^{\prime}$ which satisfy that $0 \leq \varepsilon<\boldsymbol{\varepsilon}^{\prime}$ such that $K_{s}^{\varepsilon} \not \subset K_{s}^{\varepsilon^{\prime}}$. Then, there must exist a route $k$ such that $k \in K_{s}^{\varepsilon}$ and $k \notin K_{s}^{\varepsilon^{\prime}}$. In another word, $f_{k}^{\varepsilon} \geq 0$ for all $\boldsymbol{\varepsilon}$-R-BRUE route flow patterns, and $f_{k}^{\varepsilon^{\prime}}$ can be equal to 0 for some $\boldsymbol{\varepsilon}^{\prime}$-R-BRUE route flow patterns. And this contradicts the assumption that the network has no paradoxical route.

When $\boldsymbol{\varepsilon}$ varies from zero to infinity, the minimum number of routes the largest $\boldsymbol{\varepsilon}$-URS and smallest $\boldsymbol{\varepsilon}$-URS without paradoxical route contains is the UE shortest routes when $\boldsymbol{\varepsilon}=0$; that is, $K_{l}^{0}=K_{s}^{0} \triangleq K_{\mathrm{UE}}$. The maximum number of routes the largest $\boldsymbol{\varepsilon}$-URS and smallest $\boldsymbol{\varepsilon}$-URS without paradoxical route contains is all feasible routes, meaning all feasible routes will be utilized if the indifference band is too large. Then we have $K_{\mathrm{UE}} \subseteq K_{l}^{\varepsilon} \subseteq K$ and $K_{\mathrm{UE}} \subseteq K_{s}^{\varepsilon} \subseteq K$.

Given $\boldsymbol{\varepsilon}$, the largest $\boldsymbol{\varepsilon}$-URS (defined in (7)) is a set of all used routes under $\boldsymbol{\varepsilon}$-R-BRUE set. And the smallest $\boldsymbol{\varepsilon}$-URS (defined in (8)) is a set of the used routes which must have traffic flows under all $\boldsymbol{\varepsilon}$-R-BRUE set. It is possible that some used routes for one $\boldsymbol{\varepsilon}$-R-BRUE flow pattern are not used for other flow patterns and vice versa. This necessitates the exploration of the interior structure of $\boldsymbol{\varepsilon}$-R-BRUE route set. Theorems 7 and 9 provide us with one approach of analyzing the structure of $\boldsymbol{\varepsilon}$-R-BRUE without paradoxical route by varying values of $\boldsymbol{\varepsilon}$.

\section{Generation of $\varepsilon$-R-BRUE Route Set without Paradoxical Route}

The route set is finite, while $\boldsymbol{\varepsilon}$ is treated as a continuous parameter for the time being. Starting with the UE route set when $\boldsymbol{\varepsilon}=0$, provided the network topology and the link cost functions, UE can be determined by some established 
algorithms, for example, simplicial decomposition with disaggregated (DSD) algorithm [31], gradient projection algorithm [32], or origin-based algorithm [33]. According to Theorems 7 and 9, when $\boldsymbol{\varepsilon}$ is gradually increased, more routes will be included in the largest and smallest $\boldsymbol{\varepsilon}$-URS, and we should be able to identify those used routes one by one, until all alternative routes are included. This offers the theoretical foundation for deriving different combinations of used routes by varying $\boldsymbol{\varepsilon}$ subsequently.

4.1. Definition of Critical Points in $\varepsilon-R-B R U E$. It is assumed that there are $n$ alternative routes for OD pair $\omega$; that is, $K=$ $\{1, \ldots, n\}$ and $|K|=n$, where $|K|$ is the cardinality of set $K$. Among these $n$ routes, there are $r$ shortest routes at the UE; that is, $K_{\mathrm{UE}}=\{1, \ldots, r\}$ and $\left|K_{\mathrm{UE}}\right|=r \leq n$. Below are the definitions of critical points of the largest and smallest $\varepsilon$-URS in R-BRUE without paradoxical route.

Definition 10. The largest/smallest $\varepsilon$-URS will remain the same until $\varepsilon$ reaches a special value, and we define this value as critical points of the largest/smallest $\mathcal{E}$-URS for OD pair Omega; that is,

$$
\begin{aligned}
& \varepsilon_{l, 1}^{*} \triangleq \inf _{\varepsilon>0}\left\{K_{\mathrm{UE}} \subset K_{l}^{\varepsilon}\right\} \\
& \varepsilon_{l, j}^{*} \triangleq \inf _{\varepsilon>0}\left\{K_{l}^{\varepsilon_{l, j-1}^{*}} \subset K_{l}^{\varepsilon}\right\}, \\
& \varepsilon_{l, J}^{*} \triangleq \inf _{\varepsilon>0}\left\{K_{l}^{\varepsilon}=K\right\} . \\
& \varepsilon_{s, 1}^{*} \triangleq \inf _{\varepsilon>0}\left\{K_{\mathrm{UE}} \subset K_{s}^{\varepsilon}\right\} \\
& \varepsilon_{s, i}^{*} \triangleq \inf _{\varepsilon>0}\left\{K_{s}^{\varepsilon_{s, i-1}^{*}} \subset K_{s}^{\varepsilon}\right\} ; \\
& \varepsilon_{s, I}^{*} \triangleq \inf _{\varepsilon>0}\left\{K_{s}^{\varepsilon}=K\right\},
\end{aligned}
$$

where $j=1, \ldots, J, i=1, \ldots, I$ are the unique sequences of finite critical points $\varepsilon_{l}^{*}$ and $\varepsilon_{s}^{*}$, with $\varepsilon_{l, 0}^{*}=\varepsilon_{s, 0}^{*}=0, \varepsilon_{l, J+1}^{*}=$ $\varepsilon_{s, I+1}^{*}=\infty$.

We give the physical meaning of $\varepsilon_{l}^{*}$ and $\varepsilon_{s}^{*}$. A "newly added route" of the largest $\varepsilon$-URS is defined as the route which is unavailable under $\varepsilon_{l, j-1}^{*}$ but available when $\varepsilon=\varepsilon_{l, j}^{*}$. And a "newly added route" of the smallest $\varepsilon$-URS is defined as the route which is available under $\varepsilon_{s, z}^{*}: \forall z \geq i$ for all route flow patterns, but unavailable under $\varepsilon_{s, i-1}^{*}$ for some route flow patterns. We can define their "newly added route" as $r_{j}^{*} \triangleq$ $\left\{k \in K: k \in K_{l}^{\varepsilon_{l, j}^{*}}, k \notin K_{l}^{\varepsilon_{l, j-1}^{*}}\right\}$ and $t_{i}^{*} \triangleq\left\{k \in K: k \in K_{s}^{\varepsilon_{s, i}^{*}}, k \notin\right.$ $K_{s}^{\left.\varepsilon_{s, i-1}^{*}\right\}}$, respectively. We should notice that the number of "newly added routes" may be two or more at the same time.

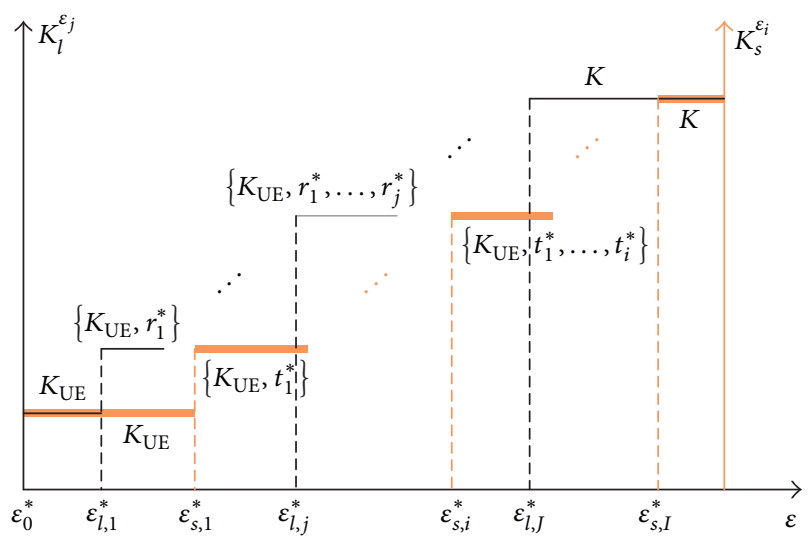

FIGURE 4: Monotonically nondecreasing property of critical points.

We use Figure 4 to more intuitively illustrate the definition of critical points of the largest and smallest $\boldsymbol{\varepsilon}$-URS in RBRUE without paradoxical route.

Clearly, from Figure 4, we can observe that the largest and smallest $\varepsilon$-URS can be described as $K_{l}^{\varepsilon}=\left\{K_{\mathrm{UE}}, K_{l}^{\varepsilon_{1}^{*}}, \ldots, K_{l}^{\varepsilon_{P}^{*}}\right\}$ and $K_{s}^{\varepsilon}=\left\{K_{\mathrm{UE}}, K_{s}^{\varepsilon_{1}^{*}}, \ldots, K_{s}^{\varepsilon_{Q}^{*}}\right\}$ with a fixed indifference band $\varepsilon$, where $\varepsilon_{l, M}^{*} \leq \varepsilon<\varepsilon_{l, M+1}^{*}, \varepsilon_{s, N}^{*} \leq \varepsilon<\varepsilon_{s, N+1}^{*}, P=\min \{M, J\}$, and $=\min \{N, I\}$.

4.2. $\varepsilon$-R-BRUE Route Set without Paradoxical Route for One $O D$ Pair. Definition 10 says that the largest and smallest $\varepsilon$ URS in R-BRUE without paradoxical route includes more routes when $\varepsilon$ increases to some critical values. Thus, the mathematical programs (MP) with equilibrium constraint can be developed to solve these critical values in largest and smallest $\varepsilon$-URS. Below is the MP equation for calculating largest $\mathcal{\varepsilon}$-URS:

$$
\begin{array}{ll}
\min & \varepsilon_{l, j} \\
\text { s.t. } & C_{k}(\mathbf{f})-\pi-\rho_{k}=0, \quad \forall k \in K, \\
& f_{k}\left(\varepsilon_{l, j}-\rho_{k}\right) \begin{cases}=0, & \text { if } \varepsilon_{l, j} \leq \rho_{k}, \quad \forall k \in K, \\
>0, & \text { o.w., }\end{cases} \\
& \sum_{k \in K} f_{k}=q, \\
& f_{k} \geq 0, \quad \forall k \in K, \\
& \rho_{k} \geq 0, \quad \forall k \in K, \\
& \sum_{\varepsilon_{l, j-1}^{*}} f_{j}<q,
\end{array}
$$

where $j=1, \ldots, J$. Equations (13a)-(13e) are to guarantee the route flow pattern is a feasible R-BRUE; (13f) tries to push a small amount of flow from the largest used route set $K_{l}^{\varepsilon_{l, j-1}^{*}}$ to some newly largest used route if $\varepsilon_{l}$ is increased a little bit. When (13a), (13b), (13c), (13d), (13e), and (13f) are solved, 


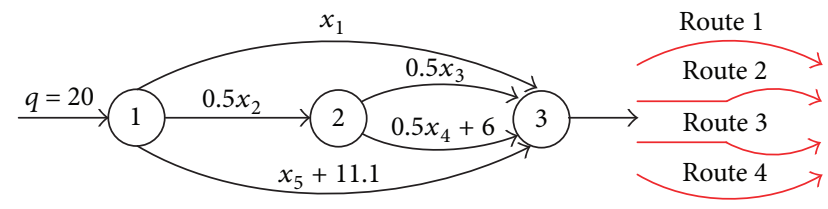

FIGURE 5: Test network and routes characteristics.

optimal solutions $\left(\mathbf{f}^{*}, \varepsilon_{l, j}^{*}, \pi^{*}\right)$ will be obtained. And the newly added route $r_{j}^{*}$ can be derived from the traffic flow $\mathbf{f}^{*}$.

For any route $i \in K$, following MP equation is used to get the smallest $\varepsilon$-URS:

$$
\begin{array}{ll}
\max & \varepsilon_{s, i} \\
\text { s.t. } & C_{k}(\mathbf{f})-\pi-\rho_{k}=0, \quad \forall k \in K, \\
& f_{k}\left(\varepsilon_{s, i}-\rho_{k}\right) \begin{cases}=0, & \text { if } \varepsilon_{s, i} \leq \rho_{k}, \quad \forall k \in K, \\
>0, & \text { o.w., }\end{cases} \\
& \sum_{k \in K} f_{k}=q, \\
& f_{k} \geq 0, \quad \forall k \in K, \\
& \rho_{k} \geq 0, \quad \forall k \in K, \\
& f_{i}=0 .
\end{array}
$$

Equations (15a)-(15e) are to guarantee the route flow pattern is a feasible R-BRUE; and (15f) is to insure that no traffic flow travels on route $i$. When $\varepsilon_{s, i}$ for all routes are worked out, $\varepsilon_{s, i}$ are sorted by their size. Then we get the form of $\varepsilon_{s, i}$ as (11). When (15a), (15b), (15c), (15d), (15e), and (15f) are solved, optimal solutions $\left(\mathbf{f}^{*}, \varepsilon_{s, i}^{*}, \pi^{*}\right)$ will be obtained. And the newly added route $t_{i}^{*}$ can be derived from the traffic flow $\mathbf{f}^{*}$.

Equations (13b), (13f), and (15b) are inequalities without equal sign. A small positive parameter $\delta$ (such as 0.01 ) is introduced to deal with this problem. And (13b), (13f), and (15b) are replaced by

$$
\begin{gathered}
f_{k}\left(\varepsilon_{l, j}-\rho_{k}\right) \begin{cases}=0, & \text { if } \varepsilon_{l, j} \leq \rho_{k}, \\
\geq \delta, & \text { o.w., }\end{cases} \\
\quad-\sum_{j \in K_{l}^{\varepsilon_{l}^{*}, j-1}} f_{j} \geq(\delta-1) q, \\
f_{k}\left(\varepsilon_{s, i}-\rho_{k}\right) \begin{cases}=0, & \text { if } \varepsilon_{s, i} \leq \rho_{k}, \\
\geq \delta, & \text { o.w. }\end{cases}
\end{gathered}
$$

Equations (13a), (13b), (13c), (13d), (13e), (13f), (15a), (15b), $(15 \mathrm{c}),(15 \mathrm{~d}),(15 \mathrm{e})$, and (15f) can be solved by GAMS software $[25,34]$.

4.3. Instance of $\boldsymbol{\varepsilon}$-R-BRUE Route Set without Paradoxical Route. The network topology of the test network, travel time functions, and traffic demand are depicted in Figure 5. The

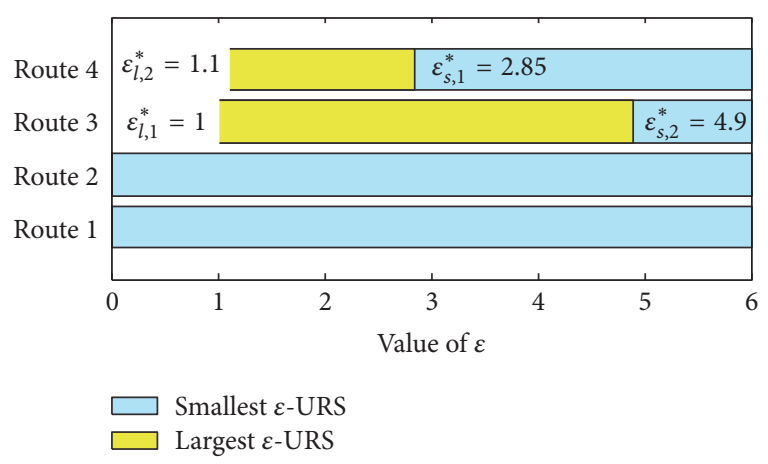

FIGURE 6: $\varepsilon$-URS under all the critical points.

network consists of 3 nodes, 5 links, and 1 OD pair. Red curves on the right indicate 4 routes. The UE is $f_{1}=10, f_{2}=10$, $f_{3}=0$, and $f_{4}=0$.

Solving (13a), (13b), (13c), (13d), (13e), (13f), (15a), (15b), (15c), (15d), (15e), and (15f), we have the largest critical values $\varepsilon_{l, 0}^{*}=0, \varepsilon_{l, 1}^{*}=1, \varepsilon_{l, 2}^{*}=1.1$, and $\varepsilon_{l, 3}^{*}=\infty$ and the smallest critical values $\varepsilon_{s, 0}^{*}=0, \varepsilon_{s, 1}^{*}=2.85, \varepsilon_{s, 2}^{*}=4.9$, and $\varepsilon_{s, 3}^{*}=\infty$. There are three cases for the largest $\varepsilon$-URS:

(1) $0 \leq \varepsilon<1: K_{l}^{\varepsilon}=\{1,2\}$, and when $\varepsilon=0$, $\mathbf{f}=$ $[10,10,0,0], \mathbf{C}=[10,10,10,11.1]$.

(2) $1 \leq \varepsilon<1.1: K_{l}^{\varepsilon}=\{1,2,3\}$, and when $\varepsilon=1, \mathbf{f}=$ $\left[10,10,0^{+}, 0\right], \mathbf{C}=[10,10,10,11.1]$.

(3) $\varepsilon \geq 1.1: K_{l}^{\varepsilon}=\{1,2,3,4\}$, and when $\varepsilon=1.1, \mathbf{f}=$ $\left[10,10,0^{+}, 0^{+}\right], \mathbf{C}=[10,10,10,11.1]$.

And there are three cases for the smallest $\mathcal{E}$-URS:

(1) $0 \leq \varepsilon<2.85: K_{s}^{\varepsilon}=\{1,2\}$, and when $\varepsilon=0$, $\mathbf{f}=$ $[10,10,0,0], \mathbf{C}=[10,10,10,11.1]$.

(2) $2.85 \leq \varepsilon<4.9: K_{s}^{\varepsilon}=\{1,2,4\}$, and when $\varepsilon=2.85$, $\mathbf{f}=\left[11.1,7.6,1.3,0^{+}\right], \mathbf{C}=[11.1,8.25,11.1,11.1]$.

(3) $\varepsilon \geq 4.9: K_{s}^{\varepsilon}=\{1,2,3,4\}$, and when $\varepsilon=4.9, \mathbf{f}=$ $\left[7.1,12,0^{+}, 0.9\right], \mathbf{C}=[7.1,12,12,12]$.

All largest and smallest $\varepsilon$-URS are also illustrated in Figure 6 . For route 3 , we can see that when the critical value $\varepsilon$ reaches 1 , route 3 will join in the used route set for some route flow patterns; and when $\varepsilon$ reaches 4.9 , route 3 will be the used route for all route flow patterns. In other words, the yellow bar in Figure 6 means the route is either the used route or unused route in different route flow patterns, while the blue bar represents the route must be a used route for all route flow patterns. If $\varepsilon$ is calibrated from empirical data as 3 , then $K_{l}^{3}=$ $\{1,2,3,4\}, K_{s}^{3}=\{1,2,4\}$. Therefore, routes 1,2 , and 4 must be the used routes, and route 3 may carry traffic flow or not. The used route can be described as $K^{3}=\{\{1,2,4\},\{1,2,3,4\}\}$.

4.4. $\varepsilon$-R-BRUE Route Set without Paradoxical Route for Multiple OD Pairs. For a network with total $W$ OD pairs, let $\varepsilon_{l, j}^{* \omega}$ and $\varepsilon_{s, i}^{* \omega}$ be the largest and smallest critical points for OD pair $\omega \in W, j=0,1, \ldots, J^{\omega}, i=0,1, \ldots, I^{\omega}$, respectively. Then, $\varepsilon_{l, j}^{*} \triangleq\left\{\varepsilon_{l, j}^{* \omega}\right\}$ and $\varepsilon_{s, i}^{*} \triangleq\left\{\varepsilon_{s, i}^{* \omega}\right\}$ are the sets of largest and smallest critical points for all OD pairs. 
For one OD pair $v \in W$, we also can use (13a), (13b), (13c), (13d), (13e), (13f), (15a), (15b), (15c), (15d), (15e), and (15f) to solve the largest and smallest critical points. The only difference is route costs need the information of route flows across all OD pairs. And route flows $\mathbf{f}^{\omega}, \omega \in W, \omega \neq v$ are parameters when calculating the critical points of $v$. Then, we modify (13a), (13b), (13c), (13d), (13e), (13f), (15a), (15b), (15c), (15d), (15e), and (15f) as follows:

$$
\begin{aligned}
& \min \varepsilon_{l, j}^{v} \\
& \text { s.t. } C_{k}^{v}(\mathbf{f})-\pi^{v}-\rho_{k}^{v}=0, \quad \forall k \in K^{v}, \\
& f_{k}^{v}\left(\varepsilon_{l, j}^{v}-\rho_{k}^{v}\right)\left\{\begin{array}{ll}
=0, & \text { if } \varepsilon_{l, j}^{v} \leq \rho_{k}^{v}, \\
>0, & \text { o.w., }
\end{array} \quad \forall k \in K^{\omega},\right. \\
& \sum_{k \in K^{\omega}} f_{k}^{\omega}=q^{\omega}, \quad \forall \omega \in W, \\
& f_{k}^{v} \geq 0, \quad \forall k \in K^{v}, \\
& \rho_{k}^{v} \geq 0, \quad \forall k \in K^{v} \text {, } \\
& \sum_{\substack{\varepsilon_{l, j-1}^{* v} \\
j \in K_{l}}} f_{j}^{v}<q^{v} \\
& \max \varepsilon_{s, i}^{v} \\
& \text { s.t. } C_{k}^{v}(\mathbf{f})-\pi^{v}-\rho_{k}^{v}=0, \quad \forall k \in K^{v} \text {, } \\
& f_{k}^{v}\left(\varepsilon_{s, i}^{v}-\rho_{k}^{v}\right)\left\{\begin{array}{ll}
=0, & \text { if } \varepsilon_{s, i}^{v} \leq \rho_{k}^{v}, \\
>0, & \text { o.w., }
\end{array} \quad \forall k \in K^{\omega},\right.
\end{aligned}
$$

$$
\begin{aligned}
& \sum_{k \in K^{\omega}} f_{k}^{\omega}=q^{\omega}, \quad \forall \omega \in W, \\
& f_{k}^{v} \geq 0, \quad \forall k \in K^{v}, \\
& \rho_{k}^{v} \geq 0, \quad \forall k \in K^{v}, \\
& f_{i}^{v}=0 .
\end{aligned}
$$

Then the same approach can be used to generate the $\boldsymbol{\varepsilon}$ R-BRUE route set without paradoxical route for multiple OD pairs.

By far we have proposed how to solve $\boldsymbol{\varepsilon}$-R-BRUE route set without paradoxical route for both single OD pair and multiple OD pairs. The following will discuss the methodology of constructing $\boldsymbol{\varepsilon}$-R-BRUE route flow set without paradoxical route.

\section{Construction of $\varepsilon$-R-BRUE Route Flow Set without Paradoxical Route}

We have already analyzed the interior structure of $\boldsymbol{\varepsilon}$-RBRUE route set without paradoxical route last section. As the indifference band gradually increases, more routes will begin to carry flows (the largest R-BRUE route set), and more routes must carry flows (the smallest R-BRUE route set). Based on this characteristic, we decompose $\boldsymbol{\varepsilon}$-R-BRUE route set into small subsets which are easier to study.

5.1. $\varepsilon$-R-BRUE Flow Set without Paradoxical Route for One OD Pair. Define a sequence of sets $F_{k}^{\varepsilon}, k=0, \ldots, M-N$, where $M$ and $N$ are the cardinalities of largest and smallest $\varepsilon$-URS. Then we assign all the traffic demands as follows:

$$
\begin{aligned}
& F_{0}^{\varepsilon} \triangleq\left\{\mathbf{f} \in F: \forall i_{1}, i_{2} \in K_{s}^{\varepsilon}: f_{i_{1}}, f_{i_{2}}>0,\left|C_{i_{1}}-C_{i_{2}}\right| \leq \varepsilon, \sum f_{i}=q ; \forall j \in K_{l}^{\varepsilon} \backslash i: f_{j}=0, C_{j}-\min C_{i} \geq \varepsilon\right\}, \\
& F_{1}^{\varepsilon} \triangleq\left\{\mathbf{f} \in F: \forall h \in K_{l}^{\varepsilon} \backslash K_{s}^{\varepsilon} ; \forall p_{1}, p_{2} \in K_{s}^{\varepsilon}, h: f_{p_{1}}, f_{p_{2}}>0,\left|C_{p_{1}}-C_{p_{2}}\right| \leq \varepsilon, \sum f_{p}=q ; \forall j \in K_{l}^{\varepsilon} \backslash p: f_{j}=0, C_{j}\right. \\
&\left.\quad-\min C_{p} \geq \varepsilon\right\}, \quad h=K_{l, 1}^{\varepsilon}, \ldots, K_{l, M-N}^{\varepsilon} \backslash K_{s}^{\varepsilon}, \\
& F_{k}^{\varepsilon} \triangleq\left\{\mathbf{f} \in F: \forall h_{1}, \ldots, h_{k} \in K_{l}^{\varepsilon} \backslash K_{s}^{\varepsilon} ; \forall p_{1}, p_{2} \in K_{s}^{\varepsilon}, h_{1}, \ldots, h_{k}: f_{p_{1}}, f_{p_{2}}>0,\left|C_{p_{1}}-C_{p_{2}}\right| \leq \varepsilon, \sum f_{p}=q ; \forall j \in K_{l}^{\varepsilon} \backslash p: f_{j}\right. \\
&\left.=0, C_{j}-\min C_{p} \geq \varepsilon\right\}, \quad h_{1}, \ldots, h_{k}: \text { every } k \text { routes in } K_{l, 1}^{\varepsilon}, \ldots, K_{l, M-N}^{\varepsilon} \text { exclude } K_{s}^{\varepsilon}, k=0, \ldots, M-N .
\end{aligned}
$$

We use a bridge network to illustrate (18) in detail. The network topology of the test network, travel time functions, and traffic demand are depicted in Figure 7. The network consists of 4 nodes, 6 links, and 1 OD pair. Red curves on the right indicate 4 routes. The indifference band $\varepsilon$ is set as 4,10 , 15,30 , and 60 .

The UE is $f_{1}=10, f_{2}=0, f_{3}=0$, and $f_{4}=10$. That is, routes 1 and 4 are utilized under UE. Substitute $K_{l}^{\varepsilon_{l, 0}^{*}}=K_{s}^{\varepsilon_{s, 0}^{*}}=$ $\{1,4\}$, route costs, and the demand into (13a), (13b), (13c), (13d), (13e), and (13f), we obtain the largest critical values $\varepsilon_{l, 0}^{*}=0, \varepsilon_{l, 1}^{*}=5, \varepsilon_{l, 2}^{*}=11.3$, and $\varepsilon_{l, 3}^{*}=\infty$. Solving (15a), (15b), (15c), (15d), (15e), and (15f), then we obtain the smallest critical values $\varepsilon_{s, 0}^{*}=0, \varepsilon_{s, 1}^{*}=17.5, \varepsilon_{s, 2}^{*}=52.5$, and $\varepsilon_{s, 3}^{*}=\infty$. There are three cases for the largest $\varepsilon$-URS:

(1) $0 \leq \varepsilon<5: K_{l}^{\varepsilon}=\{1,4\}$, and when $\varepsilon=0$, $\mathbf{f}=$ $[10,0,0,10], \mathbf{C}=[50,65,55,50]$.

(2) $5 \leq \varepsilon<11.3: K_{l}^{\varepsilon}=\{1,3,4\}$, and when $\varepsilon=5$, $\mathbf{f}=$ $\left[10,0,0^{+}, 10\right], \mathbf{C}=[50,65,55,50]$.

(3) $\varepsilon \geq 11.3: K_{l}^{\varepsilon}=\{1,2,3,4\}$, and when $\varepsilon=11.3, \mathbf{f}=$ $\left[9.26,0^{+}, 2.59,8.15\right], \mathrm{C}=[49.63,60.93,60.93,49.63]$. 


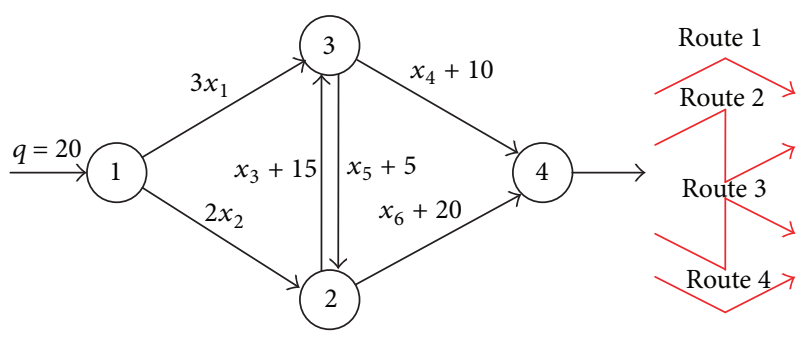

FIGURE 7: A bridge network and routes characteristics.

And there are three cases for the smallest $\varepsilon$-URS:

(1) $0 \leq \varepsilon<17.5: K_{s}^{\varepsilon}=\{1,4\}$, and when $\varepsilon=0$, $\mathbf{f}=$ $[10,0,0,10], \mathbf{C}=[50,65,55,50]$.

(2) $17.5 \leq \varepsilon<52.5: K_{s}^{\varepsilon}=\{1,3,4\}$, and when $\varepsilon=17.5$, $\mathbf{f}=\left[7.5,0,0^{+}, 12.5\right], \mathbf{C}=[40,60,57.5,57.5]$.

(3) $\varepsilon \geq 52.5: K_{s}^{\varepsilon}=\{1,2,3,4\}$, and when $\varepsilon=52.5$, $\mathbf{f}=$ $\left[17.5,0^{+}, 0^{+}, 2.5\right], \mathbf{C}=[80,80,47.5,27.5]$. follows.

Then we assign all the traffic demands with diffident $\varepsilon$ as

(1) When $\varepsilon=4, K_{l}^{4}=K_{s}^{4}=\{1,4\}$, and $M=N=2$, then $F_{0}^{4} \triangleq\left\{\mathbf{f} \in F: f_{1}, f_{4}>0,\left|C_{1}(\mathbf{f})-C_{4}(\mathbf{f})\right| \leq 4, f_{1}+f_{4}\right.$

$=20\}$.

(2) When $\varepsilon=10, K_{l}^{10}=\{1,3,4\}, K_{s}^{10}=\{1,4\}, M=3$, and $N=2$, then

$$
\begin{aligned}
F_{0}^{10} & \triangleq\left\{\mathbf{f} \in F: f_{1}, f_{4}>0,\left|C_{1}(\mathbf{f})-C_{4}(\mathbf{f})\right| \leq 10, f_{1}\right. \\
& +f_{4}=20 ; f_{3}=0, C_{3}(\mathbf{f})-\min \left\{C_{1}(\mathbf{f}), C_{4}(\mathbf{f})\right\} \\
& \geq 10\}, \\
F_{1}^{10} & \triangleq\left\{\mathbf{f} \in F: \forall i_{1}, i_{2} \in\{1,4\}, h \in\{3\} ; \forall p_{1}, p_{2}\right. \\
& \in\{1,3,4\}: f_{p_{1}}, f_{p_{2}}>0,\left|C_{p_{1}}(\mathbf{f})-C_{p_{2}}(\mathbf{f})\right| \\
& \left.\leq 10, f_{1}+f_{3}+f_{4}=20\right\} .
\end{aligned}
$$

(3) When $\varepsilon=15, K_{l}^{15}=\{1,2,3,4\}, K_{s}^{15}=\{1,4\}, M=4$, and $N=2$, then

$$
\begin{aligned}
& F_{0}^{15} \triangleq\left\{\mathbf{f} \in F: f_{1}, f_{4}>0,\left|C_{1}(\mathbf{f})-C_{4}(\mathbf{f})\right| \leq 15, f_{1}\right. \\
&+f_{4}=20 ; \forall j \in\{2,3\}: f_{j}=0, C_{j} \\
&\left.-\min \left\{C_{1}(\mathbf{f}), C_{4}(\mathbf{f})\right\} \geq 15\right\}, \\
& F_{1}^{15} \triangleq\left\{\mathbf{f} \in F: \forall h \in\{2,3\} ; \forall p_{1}, p_{2}\right. \\
& \quad \in\{1,4\}, h: f_{p_{1}}, f_{p_{2}}>0,\left|C_{p_{1}}(\mathbf{f})-C_{p_{2}}(\mathbf{f})\right| \\
& \quad \leq 15, \sum f_{p}=20 ; \forall j \in\{1,2,3,4\} \backslash p: f_{j}=0, C_{j} \\
&\left.\quad-\min C_{p} \geq 15\right\}, \quad h=2,3,
\end{aligned}
$$

$$
\begin{aligned}
F_{2}^{15} & \triangleq\left\{\mathbf{f} \in F: \forall h_{1}, h_{2} \in\{2,3\} ; \forall p_{1}, p_{2}\right. \\
& \in\{1,2,3,4\}: f_{p_{1}}, f_{p_{2}}>0,\left|C_{p_{1}}(\mathbf{f})-C_{p_{2}}(\mathbf{f})\right| \\
& \left.\leq 15, \sum f_{p}=20\right\} .
\end{aligned}
$$

(4) When $\varepsilon=30, K_{l}^{30}=\{1,2,3,4\}, K_{s}^{30}=\{1,3,4\}, M=4$, and $N=3$, then

$$
\begin{aligned}
& F_{0}^{30} \triangleq\left\{\mathbf{f} \in F: \forall i_{1}, i_{2} \in\{1,3,4\}: f_{i_{1}}, f_{i_{2}}\right. \\
&>0,\left|C_{i_{1}}(\mathbf{f})-C_{i_{2}}(\mathbf{f})\right| \leq 30, f_{1}+f_{3}+f_{4}=20 ; f_{2} \\
&\left.=0, C_{2}(\mathbf{f})-\min C_{i} \geq 30\right\}, \\
& F_{1}^{30} \triangleq\left\{\mathbf{f} \in F: h \in\{2\} ; \forall p_{1}, p_{2} \in\{1,2,3,4\}: f_{p_{1}}, f_{p_{2}}\right. \\
&>0,\left|C_{p_{1}}(\mathbf{f})-C_{p_{2}}(\mathbf{f})\right| \leq 30, f_{1}+f_{2}+f_{3}+f_{4} \\
&=20\} .
\end{aligned}
$$

(5) When $\varepsilon=60, K_{l}^{60}=K_{s}^{60}=\{1,2,3,4\}$, and $M=N=$ 4 , then

$$
\begin{aligned}
F_{0}^{60} & \triangleq\left\{\mathbf{f} \in F: \forall i_{1}, i_{2} \in\{1,2,3,4\}: f_{i_{1}}, f_{i_{2}}\right. \\
& >0,\left|C_{i_{1}}(\mathbf{f})-C_{i_{2}}(\mathbf{f})\right| \leq 60, f_{1}+f_{2}+f_{3}+f_{4} \\
& =20\} .
\end{aligned}
$$

For convenience, we only show the R-BRUE solution sets with $\varepsilon=4,10,15$ in Figure 8. Due to the flow conservation of the fixed demand, its R-BRUE solution sets can be characterized by routes 1,3 , and 4 in Figures $8(\mathrm{~b})$ and $8(\mathrm{c})$. Figure 8(a) is composed of a 2-route green subset; Figure 8(b) is composed of a 2-route green subset and 3-route yellow subset; Figure $8(\mathrm{c})$ is composed of a 2 -route green subset, 3 -route yellow subset, and 4-route blue subset. The magenta legend denotes that the solutions do not satisfy $\varepsilon$-R-BRUE.

From Figure 8, we can see that (1) when $\varepsilon=15$, in $F_{0}^{15}$ (green subset), only routes 1 and 4 carry flow, so the subset is a line. In $F_{1}^{15}$ (yellow subset), route 3 begins to carry flow and $f_{3}>0$. In $F_{2}^{15}$ (blue subset), route 2 begins to carry flow and $f_{2}>0$; (2) the solution set is bounded; this is because $F_{\text {R-BRUE }}^{\varepsilon} \subset F: F \triangleq\left\{\mathbf{f}: \mathbf{f} \geq 0, \sum_{k \in K^{\omega}} f_{k}^{\omega}=q^{\omega}, \forall \omega \in W\right\}$, while its closeness cannot be guaranteed due to $C_{k}^{\omega}(\mathbf{f})<$ $\min _{i \in K^{\omega}} C_{i}^{\omega}(\mathbf{f})+\varepsilon^{\omega} \Rightarrow f_{k}^{\omega}>0$. In Figure 8(b), the travel time of the UE solution $\mathbf{f}_{\mathrm{UE}}=[10,0,0,10]$ on the magenta line is $\mathrm{C}_{\mathrm{UE}}=[50,65,55,50]$, and $C_{3}=55<50+15$, so $f_{3}$ should be greater than 0 in R-BRUE model; hence $\mathbf{C}_{\mathrm{UE}}$ do not satisfy 10 R-BRUE; (3) without considering the boundary point of $\mathcal{E}$-RBRUE, the solution set is monotonically nondecreasing with the increase of $\varepsilon$; and (4) the subset is not necessarily convex even though the link performance function is affine linear; this is because the constraint condition of $C_{j}-\min C_{i} \geq$ $\varepsilon$ is nonconvex. This property can be seen in the yellow block which is nonconvex in Figure 8(c). Hence, we cannot guarantee the convexity of $\varepsilon$-R-BRUE solution set. 


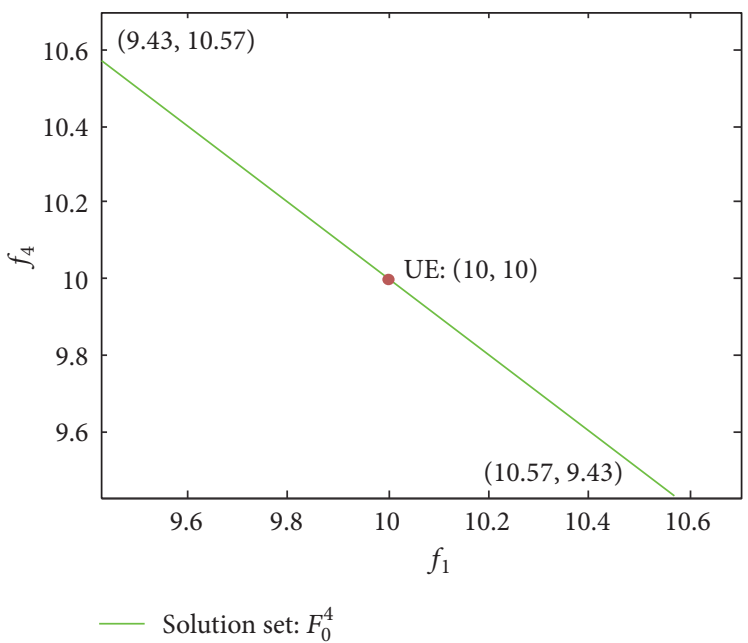

(a) R-BRUE solution set with $\varepsilon=4$

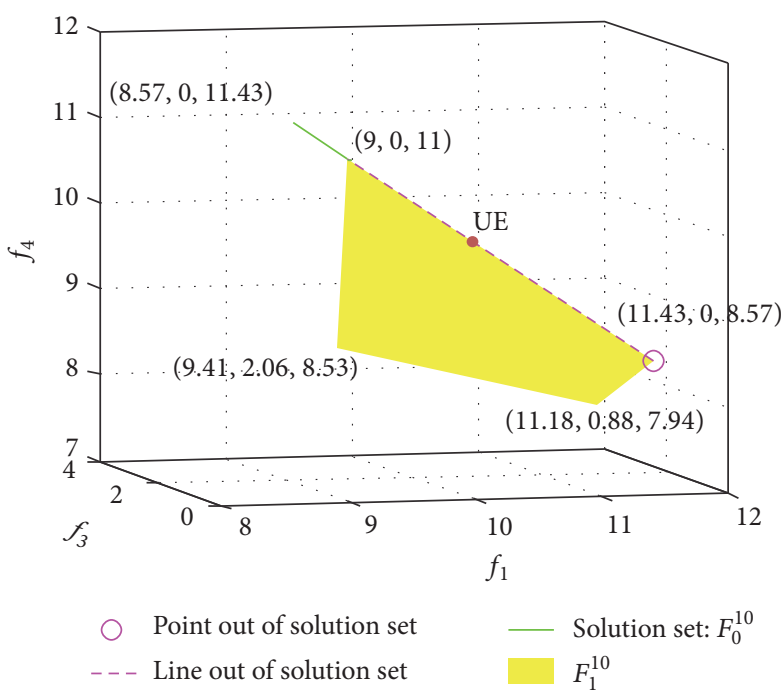

(b) R-BRUE solution set with $\varepsilon=10$

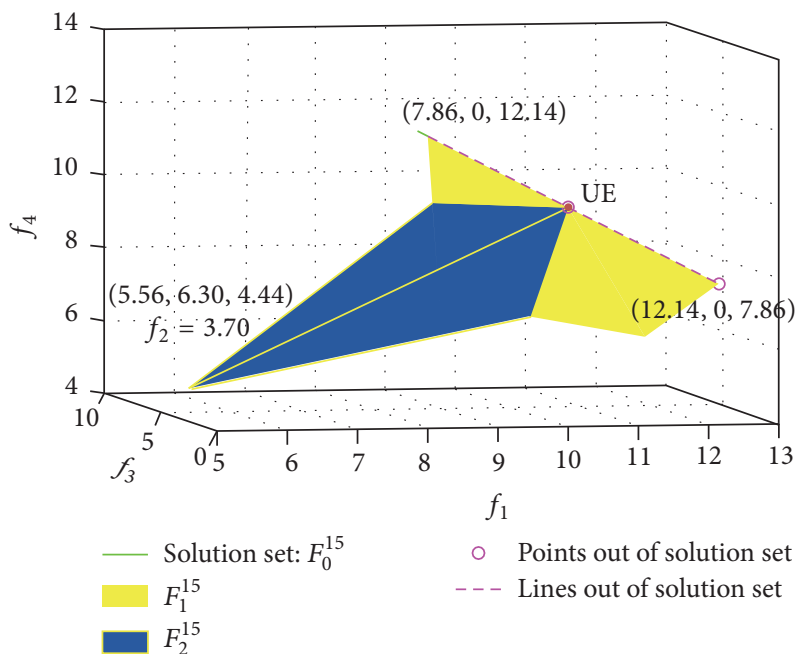

(c) R-BRUE solution set with $\varepsilon=15$

FIGURE 8: R-BRUE solution sets illustration.

5.2. $\boldsymbol{\varepsilon}$-R-BRUE Flow Set without Paradoxical Route for Multiple $O D$ Pairs. In the last section, we obtain $\boldsymbol{\varepsilon}$-R-BRUE route set without paradoxical route for multiple OD pairs. Then, it is not difficult to generalize the methodology of constructing
$\boldsymbol{\varepsilon}$-R-BRUE flow set without paradoxical route for a single OD pair to multiple OD pairs. We assign all the traffic demands to the routes as follows:

$$
\begin{aligned}
F_{0}^{\varepsilon} & \triangleq\left\{\mathbf{f} \in F: \forall \omega \in W, \forall i_{1}^{\omega}, i_{2}^{\omega} \in K_{s}^{\varepsilon^{\omega}}: f_{i_{1}^{\omega}}, f_{i_{2}^{\omega}}>0,\left|C_{i_{1}^{\omega}}-C_{i_{2}^{\omega}}\right| \leq \varepsilon^{\omega}, \sum f_{i^{\omega}}=q^{\omega} ; \forall j^{\omega} \in K_{l}^{\varepsilon^{\omega}} \backslash i^{\omega}: f_{j^{\omega}}=0, C_{j^{\omega}}\right. \\
& \left.-\min C_{i^{\omega}} \geq \varepsilon^{\omega}\right\}, \\
F_{k}^{\varepsilon} & \triangleq\left\{\mathbf{f} \in F: \forall \omega \in W, \forall h_{1}^{\omega}, \ldots, h_{k^{\omega}}^{\omega} \in K_{l}^{\varepsilon^{\omega}} \backslash K_{s}^{\varepsilon^{\omega}} ; \forall p_{1}^{\omega}, p_{2}^{\omega} \in K_{s}^{\varepsilon^{\omega}}, h_{1}^{\omega}, \ldots, h_{k^{\omega}}^{\omega}: f_{p_{1}^{\omega}}, f_{p_{2}^{\omega}}>0,\left|C_{p_{1}^{\omega}}-C_{p_{2}^{\omega}}\right| \leq \varepsilon^{\omega}, \sum f_{i^{\omega}}\right. \\
& \left.=q^{\omega} ; \forall j^{\omega} \in K_{l}^{\varepsilon^{\omega}} \backslash p^{\omega}: f_{j^{\omega}}=0, C_{j^{\omega}}-\min C_{p^{\omega}} \geq \varepsilon^{\omega}\right\},
\end{aligned}
$$$$
h_{1}^{\omega}, \ldots, h_{k^{\omega}}^{\omega} \text { : every } k^{\omega} \text { routes in } K_{l, 1}^{\varepsilon^{\omega}}, \ldots, K_{l, M-N}^{\varepsilon^{\omega}} \text { exclude } K_{s}^{\varepsilon^{\omega}}, \sum k^{\omega}=k, k=0, \ldots, M-N \text {. }
$$ 


\section{Conclusions}

In this paper, a boundedly rational user equilibrium model with restricted unused routes (R-BRUE) was proposed. This new model assumes that travel cost of all used route is less than or equal to the sum of given indifference band and the shortest route cost; meanwhile, the travel cost of the unused route is greater than or equal to the sum of indifference band and the shortest route cost. The mathematical formulation of the proposed model was then established.

Before constructing the R-BRUE flow set, largest and smallest used route sets were explored first. As the value of the indifference band increases, some routes which were not utilized before will be taken, and thus the route set that contains the equilibrium flow was named as the largest used route set. As the value of the indifference band increases, some routes which must be utilized for all R-BRUE flow patterns will be taken, and this route set was defined as the smallest used route set. Paradoxical route is defined as that with the increasing of OD demand; the UE flow on the paradoxical route increases first and then decreases. The monotonically nondecreasing property of largest and smallest used route sets without paradoxical route is proved.

The critical values of the indifference band to augment the largest and smallest used route sets can be identified by sequentially solving a class of mathematical programs. After the largest and smallest used route sets are obtained, the whole R-BRUE flow set can be obtained by assigning all traffic demands to the corresponding generated route set.

The proposed model is appealing in modeling realistic travel behavior. But due to the nonconvexity of the feasible region, it is difficult to get the solution using mathematical programming method, and our method which is separation of the solution sets takes heavy computational burdens. In future research, we will study the solution algorithm and then apply the built model to real traffic network, such as the autonomous vehicles. Also, it is worthwhile to extend the proposed model to the network design problem, such as enhancing capacities of the established links, congestion pricing, and adding new links to an existing road network.

\section{Competing Interests}

The authors declare that they have no competing interests.

\section{Acknowledgments}

This research is supported by the National Natural Science Foundation of China (no. 51578150 and no. 51378119), the Scientific Research Foundation of Graduate School of Southeast University (no. YBJJ1679), the Fundamental Research Funds for the Central Universities and the Research Innovation Program for College Graduates of Jiangsu Province (no. KYLX15_0150), and the China Scholarship Council (CSC) Program sponsored by the Ministry of Education in China.

\section{References}

[1] J. G. Wardrop, "Road paper. Some theoretical aspects of road traffic research," ICE Proceedings: Engineering Divisions. Thomas Telford, vol. 1, no. 3, pp. 325-362, 1952.
[2] C. F. Daganzo and Y. Sheffi, "On stochastic models of traffic assignment," Transportation Science, vol. 11, no. 3, pp. 253-274, 1977.

[3] M. S. Ramming, Network Knowledge and Route Choice, Massachusetts Institute of Technology, 2001.

[4] S. Bekhor, M. E. Ben-Akiva, and M. S. Ramming, "Evaluation of choice set generation algorithms for route choice models," Annals of Operations Research, vol. 144, pp. 235-247, 2006.

[5] C. G. Prato and S. Bekhor, "Applying branch-and-bound technique to route choice set generation," Transportation Research Record, vol. 1985, pp. 19-28, 2006.

[6] S. Zhu, The Roads Taken: Theory and Evidence on Route Choice in the Wake of The I-35W Mississippi River Bridge Collapse and Reconstruction, University of Minnesota, 2010.

[7] H. S. Mahmassani and G.-L. Chang, "On boundedly rational user equilibrium in transportation systems," Transportation Science, vol. 21, no. 2, pp. 89-99, 1987.

[8] H. S. Mahmassani and R. Jayakrishnan, "System performance and user response under real-time information in a congested traffic corridor," Transportation Research Part A: General, vol. 25, no. 5, pp. 293-307, 1991.

[9] R. Jayakrishnan, H. S. Mahmassani, and T.-Y. Hu, "An evaluation tool for advanced traffic information and management systems in urban networks," Transportation Research Part C, vol. 2, no. 3, pp. 129-147, 1994.

[10] T.-Y. Hu and H. S. Mahmassani, "Day-to-day evolution of network flows under real-time information and reactive signal control," Transportation Research Part C: Emerging Technologies, vol. 5, no. 1, pp. 51-69, 1997.

[11] H. S. Mahmassani and Y.-H. Liu, "Dynamics of commuting decision behaviour under advanced traveller information systems," Transportation Research Part C: Emerging Technologies, vol. 7, no. 2-3, pp. 91-107, 1999.

[12] K. K. Srinivasan and H. S. Mahmassani, "Role of congestion and information in trip-makers' dynamic decision processes: experimental investigation," Transportation Research Record, vol. 1676, pp. 44-52, 1999.

[13] R. Chen and H. S. Mahmassani, "Travel time perception and learning mechanisms in traffic networks," Transportation Research Record, vol. 1894, pp. 209-221, 2004.

[14] H. A. Simon, Models of Man: Social and Rational; Mathematical Essays on Rational Human Behavior in Society Setting, John Wiley \& Sons, New York, NY, USA, 1957.

[15] M. Sivak, "How common sense fails us on the road: contribution of bounded rationality to the annual worldwide toll of one million traffic fatalities," Transportation Research Part F: Traffic Psychology and Behaviour, vol. 5, no. 4, pp. 259-269, 2002.

[16] C. J. Khisty and T. Arslan, "Possibilities of steering the transportation planning process in the face of bounded rationality and unbounded uncertainty," Transportation Research Part C: Emerging Technologies, vol. 13, no. 2, pp. 77-92, 2005.

[17] J. L. Gifford and C. Checherita, "Bounded rationality and transportation behavior: lessons for public policy," Transportation Research Board 86th Annual Meeting 07-2451, 2007.

[18] V. Cantillo, B. Heydecker, and J. de Dios Ortúzar, "A discrete choice model incorporating thresholds for perception in attribute values," Transportation Research Part B: Methodological, vol. 40, no. 9, pp. 807-825, 2006.

[19] V. Cantillo, J. De Dios Ortúzar, and H. C. W. L. Williams, "Modeling discrete choices in the presence of inertia and serial correlation," Transportation Science, vol. 41, no. 2, pp. 195-205, 2007. 
[20] G. Marsden, K. T. Frick, A. D. May, and E. Deakin, "Bounded rationality in policy learning amongst cities: lessons from the transport sector," Environment and Planning A, vol. 44, no. 4, pp. 905-920, 2012.

[21] W. Y. Szeto and H. K. Lo, "Dynamic traffic assignment: properties and extensions," Transportmetrica, vol. 2, no. 1, pp. 31-52, 2006.

[22] Y. Lou, Y. Yin, and S. Lawphongpanich, "Robust congestion pricing under boundedly rational user equilibrium," Transportation Research Part B: Methodological, vol. 44, no. 1, pp. 1528, 2010.

[23] X. Guo and H. X. Liu, "Bounded rationality and irreversible network change," Transportation Research Part B: Methodological, vol. 45, no. 10, pp. 1606-1618, 2011.

[24] X. Guo, "Toll sequence operation to realize target flow pattern under bounded rationality," Transportation Research Part B: Methodological, vol. 56, pp. 203-216, 2013.

[25] X. Di, H. X. Liu, J.-S. Pang, and X. J. Ban, "Boundedly rational user equilibria (BRUE): mathematical formulation and solution sets," Transportation Research Part B: Methodological, vol. 57, pp. 300-313, 2013.

[26] X. Di, X. He, X. Guo, and H. X. Liu, "Braess paradox under the boundedly rational user equilibria," Transportation Research Part B: Methodological, vol. 67, pp. 86-108, 2014.

[27] X. Di, Boundedly Rational User Equilibrium: Models and Applications, University of Minnesota, 2014.

[28] K. Han, W. Y. Szeto, and T. L. Friesz, "Formulation, existence, and computation of boundedly rational dynamic user equilibrium with fixed or endogenous user tolerance," Transportation Research Part B: Methodological, vol. 79, pp. 16-49, 2015.

[29] H. S. Mahmassani and R.-C. Jou, "Transferring insights into commuter behavior dynamics from laboratory experiments to field surveys," Transportation Research Part A: Policy and Practice, vol. 34, no. 4, pp. 243-260, 2000.

[30] P. Patriksson, The Traffic Assignment Problem: Models and Methods, 1994.

[31] T. Larsson and M. Patriksson, "Simplicial decomposition with disaggregated representation for the traffic assignment problem," Transportation Science, vol. 26, no. 1, pp. 4-17, 1992.

[32] R. Jayakrishnan, W. T. Tsai, J. N. Prashker, and S. Rajadhyaksha, A Faster Path-based Algorithm for Traffic Assignment, University of California Transportation Center, 1994.

[33] H. Bar-Gera, Origin-Based Algorithms for Transportation Network Modeling, 1999.

[34] E. Rosenthal, GAMS-A User's Guide, GAMS Development Corporation, 2008. 


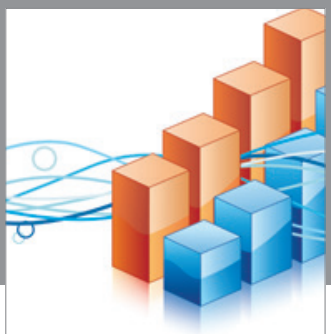

Advances in

Operations Research

vatem alat4

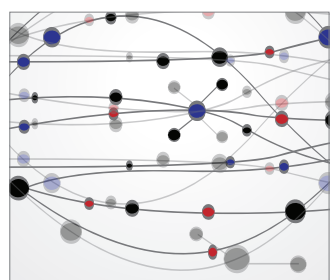

\section{The Scientific} World Journal
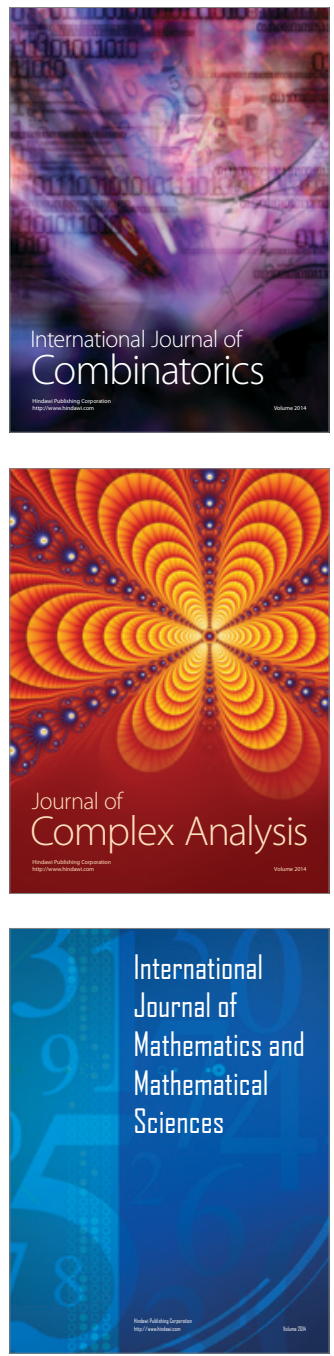
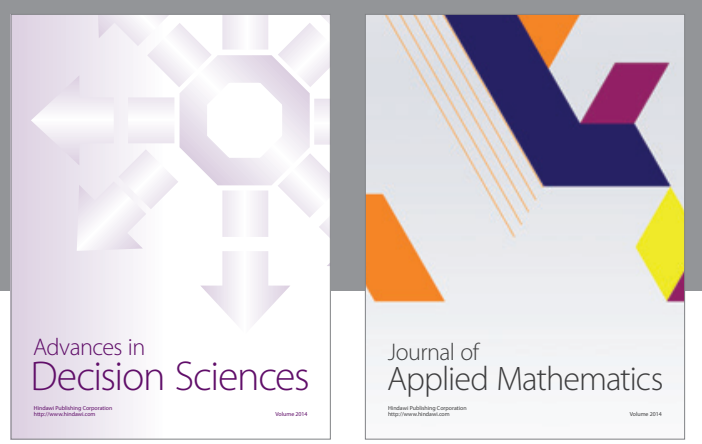

Algebra

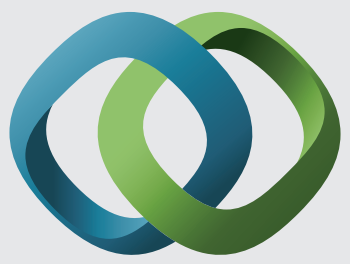

\section{Hindawi}

Submit your manuscripts at

http://www.hindawi.com
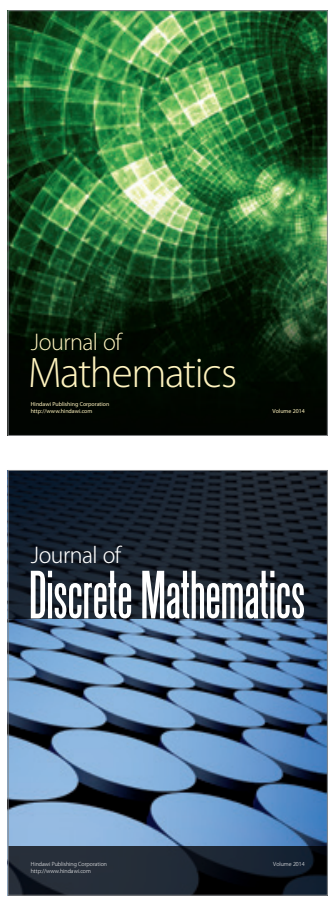

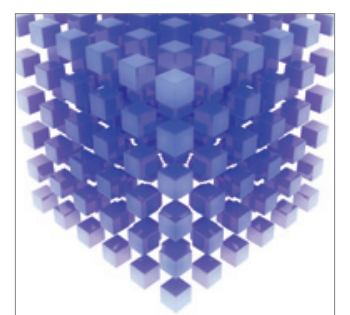

Mathematical Problems in Engineering
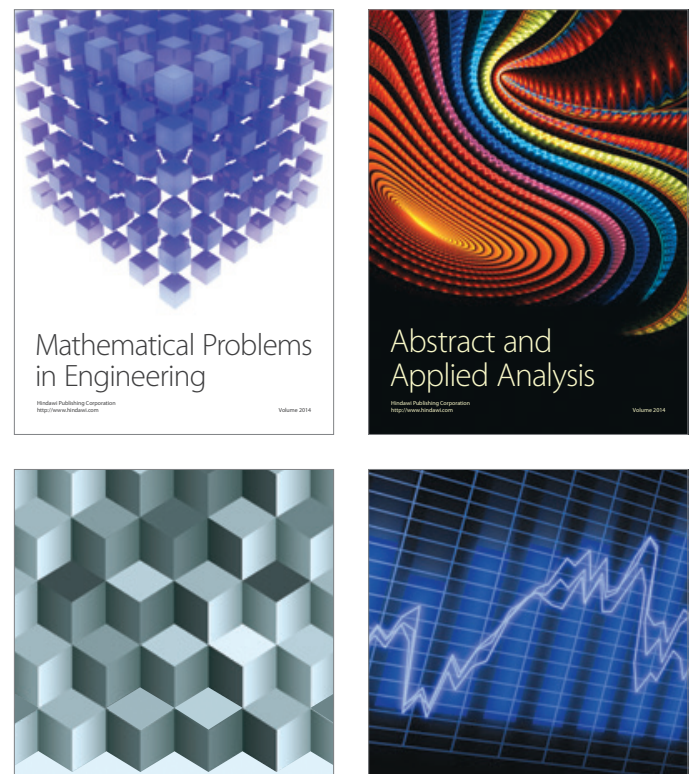

Journal of

Function Spaces

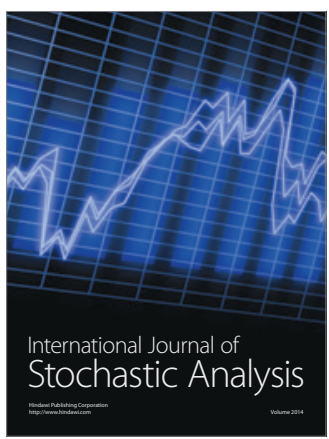

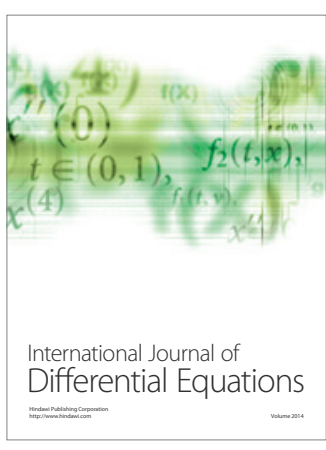
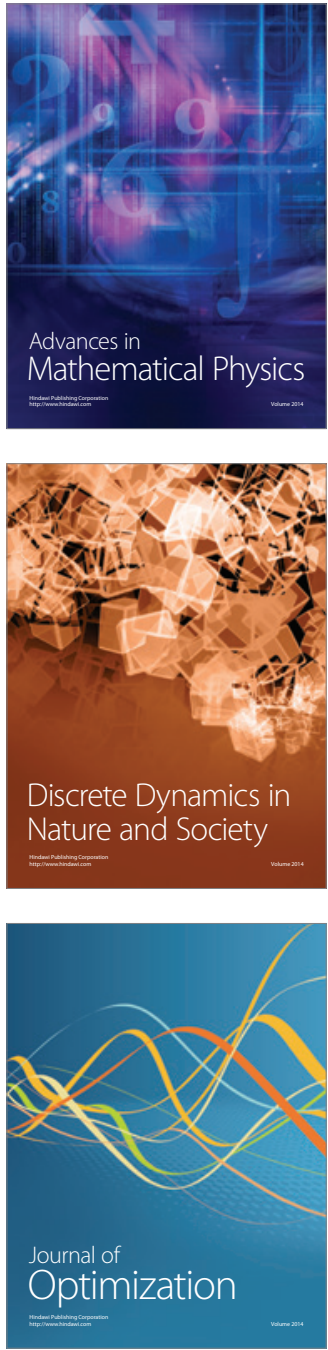